\title{
Fault Diagnosis Reasoning Algorithm Based on Multi-signal Model
}

\author{
LIU Li-jia ${ }^{1, *}, H U$ Jian-wang ${ }^{1}$, and SUN Hui-xian ${ }^{1}$ \\ ${ }^{1}$ Army Engineering University, Department of Electronics and Optical Engineering, 050003 Shijiazhuang, China
}

\begin{abstract}
The multi-signal model is modeled in the fault space and combines the structural model and the dependent model of the system. The modeling work is easy to implement in different layers and is very suitable for fault modeling and fault diagnosis of the Command and control system. After the model is established, how to meet the requirements of fault coverage and fault isolation rate, fault diagnosis reasoning algorithm is particularly important. The system dependency matrix is obtained by establishing a multi-signal model. Based on this, the fault diagnosis reasoning algorithm is studied. The fault diagnosis of Apollo spacecraft before launch is taken as an example to verify the effectiveness of fault diagnosis inference algorithm based on multi-signal model.
\end{abstract}

\section{Introduction}

With the progress of manufacturing technology, the space utilization rate is increasing day by day. The integration and miniaturization have become the development trends of accusation equipment. This largely limits the access of test points. Therefore, the increasing failure modes of equipment, and the decrease of accessibility of monitoring points lead to the decrease of equipment observability. These two aspects make fault diagnosis of accusing equipment more and more challenging[1].

Based on the multi-signal model method, this paper generates the correlation matrix and performs fault diagnosis reasoning. The conclusion is reached. The method is validated by taking the fault diagnosis of Apollo spacecraft as an example.

\section{Fault diagnosis based on multi-signal model}

\subsection{The characteristics of multi-signal model}

Multi-signal flow graph model [2], referred to as the multi-signal model, first proposed by the Connecticut University Deb and Professor Pattipati et al proposed and based on the development of commercial software TEAMS[3](testability engineering and maintenance system). It has the following characteristics [4-5]:

(1) Multi-signal model in the form of structural model is based on the coverage of the dependent model. The model is close to the block diagram of the system, which provides convenient modeling, simple verification and better interpretation of the fault.
(2) Multi-signal model for failure mode modeling needs only knowledge of functional structure. Compared with qualitative or quantitative models, multi-signal model has the advantages of fast modeling speed, convenient updating and maintenance, fast diagnosis and reasoning, and is more suitable for large and complex systems.

(3) Multi-signal model distinguishes between functional faults and global faults, which can better reflect the actual situation of complex systems.

\subsection{Fault diagnosis flow based on multi-signal model}

The specific process is as follows:

1. Using the FMECA information during the design phase, analyze the possible failure modes at each level and the influence signal for each failure mode.

2. Analyze the system block diagram, structure diagram and other information, the use of related software, such as TEAMS, the establishment of multisignal model at all levels, adding test and signal, the establishment of a complete system of multi-signal model.

3. Using multi-signal model to generate the system's D matrix (also known as the diagnostic matrix or dependency matrix or correlation matrix).

4. Observing and recording real-time test signal, and signal processing, the output of the test vector data, as the basis for subsequent troubleshooting.

5. Perform troubleshooting. The test vector and D matrix as the input of diagnostic reasoning, the output is the result of the diagnosis, that is, the cause of the fault.

\footnotetext{
* Corresponding author: 282602421@qq.com
} 


\subsection{Multi-signal model matrix representation}

Multi-signal model method through the graphical modeling method, the system design structure diagram, expert experience and other aspects of knowledge, the final core output is fault-test correlation matrix, that is, D matrix (dependency matrix).

The general form is

$$
D_{m \times n}=\overbrace{\left[\begin{array}{cccc}
d_{11} & d_{12} & \cdots & d_{1 n} \\
d_{21} & d_{22} & \cdots & d_{2 n} \\
\vdots & \vdots & \cdots & \vdots \\
d_{m 1} & d_{m 2} & \cdots & d_{m n}
\end{array}\right]}^{\text {test: }, t_{2}, \cdots, t_{n}} \text { fault: } f_{1}, f_{2}, \cdots, f_{m}
$$

Among them, the row vector represents the failure of the module can be tested to detect the situation. If there are all zero lines, it indicates that the current test can not detect the faulty module; if all the two vector values are the same, it indicates that the current test can not distinguish the two faulty modules.

The column vectors represent the conditions under which tests can detect faults. If all zero columns, it means that this test can not detect the faulty module, which is an invalid test; if all the two vector values are the same, it indicates that there is a redundancy test.

\section{Diagnostic reasoning basic algorithm}

\section{1 diagnostic reasoning description}

Diagnostic reasoning process is based on the system fault-test correlation matrix, based on real-time test values generated by the test vector, the use of appropriate diagnostic reasoning operation, the final diagnosis [6]。

The purpose of diagnostic reasoning is to classify each unknown module into three categories as normal module, fault module and suspicious module by reasoning algorithm, and present it as the diagnosis result. The basic process of reasoning is: initialize all the modules to unknown module $\mathrm{U}$; if it can detect a module's test fails, then the module is a suspicious module, remove it from the unknown module; if it can detect the test of a module, The module is a normal module, which is removed from the suspicious module and the unknown module. The non-passed test items are used to compare the suspicious modules, and the faulty modules are determined according to the isolation precision requirements. Finally, the modules are diagnosed into three categories, that is, the faulty module,
Normal module, suspicious module In this algorithm, it is considered that the correlation matrix does not have all zero lines, that is, there is no undetectable fault, and the final diagnosis conclusion does not have an unknown module.

\subsection{Diagnostic reasoning basic algorithm}

In order to facilitate the description of the algorithm, the following definitions are defined: $A$ (all) is a set of all modules, $F$ (fault) is a set of faulty modules, $N$ (normal) is a set of normal modules, $U$ (uncertainty) is a set of suspicious modules, $K$ (unknown)is the set of unknown modules, $M_{p}$ is the set of modules passed the test, and $M_{n}$ is the set of failed modules tested.

The algorithm is as follows:

Step 1 initialization, all module status is unknown, namely $K=A, F=\varnothing, N=\emptyset, U=\varnothing$.

Step 2 Process failed tests.

The set of modules detected by the failed test $M_{n}$ is added to the suspect module set $U, U \leftarrow U \cup M_{n}$.

Step 3 Process the passed test.

(1) Add the module set $M_{p}$ detected by the passed test to the normal module set $N, N \leftarrow N \cup M_{n}$.

(2) Remove the normal module set $M_{p} \cap M_{n}$ from suspect module $U, U \leftarrow U-M_{p} \cap M_{n}$.

Step 4 Determine the faulty module set.

(1) Calculate the number of failed tests corresponding to each module in the suspect module set $U$.

(2) Compare the above calculated value, according to the requirements of fault isolation accuracy, determine the most likely fault module set $M_{f}$, added to the fault module set $F, F \leftarrow F \cup M_{f}$.

(3) The faulty module set is removed from the suspicious module set $U, U \leftarrow U-M_{f}$.

\section{Diagnostic reasoning instance verification}

The following takes Apollo spacecraft detection correlation matrix before launch as an example to discuss the effectiveness of diagnostic reasoning algorithm. The data can be found in [7]. In this example, there are 15 tests and 10 possible fault modules. The correlation matrix is shown in Table 1.

Table 1, Apollo spacecraft before launch failure - test correlation matrix

\begin{tabular}{|c|c|c|c|c|c|c|c|c|c|c|c|c|c|c|c|c|}
\hline \multirow{2}{*}{$f / t$} & & \multicolumn{15}{|c|}{ test } \\
\hline & & $\mathrm{t} 1$ & $\mathrm{t} 2$ & t3 & $\mathrm{t} 4$ & $\mathrm{t} 5$ & t6 & $\mathrm{t} 7$ & t8 & t9 & $\mathrm{t} 10$ & $\mathrm{t} 11$ & $\mathrm{t} 12$ & $\mathrm{t} 13$ & $\mathrm{t} 14$ & $\mathrm{t} 15$ \\
\hline \multirow{5}{*}{ Module } & f1 & 0 & 0 & 0 & 1 & 0 & 0 & 0 & 1 & 0 & 1 & 1 & 1 & 1 & 0 & 0 \\
\hline & $\mathrm{f} 2$ & 0 & 0 & 1 & 0 & 1 & 0 & 0 & 0 & 0 & 0 & 1 & 1 & 0 & 1 & 0 \\
\hline & f3 & 0 & 0 & 0 & 0 & 1 & 1 & 1 & 0 & 1 & 1 & 1 & 1 & 0 & 0 & 1 \\
\hline & $\mathrm{f} 4$ & 0 & 1 & 0 & 0 & 0 & 1 & 1 & 0 & 0 & 0 & 0 & 0 & 0 & 1 & 1 \\
\hline & f5 & 0 & 1 & 0 & 1 & 0 & 1 & 1 & 1 & 1 & 1 & 0 & 0 & 1 & 1 & 0 \\
\hline
\end{tabular}




\begin{tabular}{clllllllllllllll} 
f6 & 0 & 0 & 0 & 1 & 1 & 0 & 0 & 1 & 1 & 1 & 0 & 1 & 1 & 1 & 1 \\
$\mathrm{f} 7$ & 1 & 0 & 0 & 1 & 1 & 0 & 0 & 0 & 0 & 0 & 0 & 1 & 0 & 1 & 1 \\
$\mathrm{f} 8$ & 1 & 1 & 1 & 0 & 0 & 1 & 1 & 0 & 1 & 1 & 1 & 0 & 0 & 1 & 0 \\
$\mathrm{f} 9$ & 1 & 0 & 0 & 1 & 1 & 0 & 0 & 0 & 0 & 0 & 0 & 1 & 0 & 1 & 1 \\
$\mathrm{f10}$ & 1 & 1 & 1 & 1 & 0 & 0 & 1 & 0 & 0 & 0 & 1 & 0 & 1 & 1 & 0 \\
\hline
\end{tabular}

Based on diagnostic reasoning algorithm, write Matlab processing program. The verification experiments are carried out under the single fault assumption and multiple fault assumption respectively.

The assumption of a single fault assumes that only one module fails at a detection time. The following tests are conducted: Suppose a system fault occurs, and input its corresponding row vector into the diagnostic program as the theoretical test vector, and record the diagnosis reasoning conclusion and running consumption Time. The experimental results are shown in Table 2.

Table 2, Single fault hypothesis fault diagnosis inference results

\begin{tabular}{cccc}
\hline Fault status & Failed test & Fault module & $\begin{array}{c}\text { Diagnostic reasoning time } \\
(\mathrm{s})\end{array}$ \\
\hline 1 & $4,8,10,11,12,13$ & 1 & 0.006 \\
2 & $3,5,11,12,14$ & 2 & 0.006 \\
3 & $5,6,7,9,10,11,12,15$ & 3 & 0.006 \\
4 & $2,6,7,14,15$ & 4 & 0.006 \\
5 & $2,4,6,7,8,9,10,13,14$ & 5 & 0.006 \\
6 & $4,5,8,9,10,12,13,14,15$ & 6 & 0.006 \\
7 & $1,4,5,8,11,13,15$ & 7 & 0.006 \\
8 & $12,3,6,7,9,10,11,14$ & 8 & 0.006 \\
9 & $1,4,5,12,14,15$ & 9 & 0.006 \\
10 & $1,2,3,4,7,11,13,14$ & 10 & 0.006 \\
\hline
\end{tabular}

fault coverage rate of $100 \%$, fault isolation rate of $100 \%$, the average diagnostic reasoning time is $0.006 \mathrm{~s}$

The assumption of multiple faults assumes that a system may have multiple modules that fail at a certain detection time. Experiments are conducted in the case of a double fault. Suppose that any two modules in the system fail, and the corresponding elements of the two row vectors are Or operation. The new vector as the theoretical test vectors enter the diagnostic program, documenting diagnostic reasoning conclusions and running time-consuming. The experimental results are shown in Table 3.

Table 3, Multiple faults assumptions troubleshooting reasoning results (section)

\begin{tabular}{ccccc}
\hline \multirow{2}{*}{$\begin{array}{c}\text { Fault } \\
\text { status }\end{array}$} & Failed test & \multicolumn{2}{c}{$\begin{array}{c}\text { Diagnostic reasoning } \\
\text { conclusion }\end{array}$} & $\begin{array}{c}\text { Diagnostic } \\
\text { reasoning time (s) }\end{array}$ \\
\cline { 3 - 4 } & $3,4,5,8,10,11,12,13,14$ & $\begin{array}{c}\text { Fault } \\
\text { module }\end{array}$ & $\begin{array}{c}\text { Suspicious } \\
\text { module }\end{array}$ & 0.006 \\
\hline 1,2 & $4,5,6,7,8,9,10,11,12,13,15$ & 1,2 & & 0.006 \\
1,3 & $2,4,6,7,8,10,11,12,13,14,15$ & 1,4 & & 0.006 \\
1,4 & $2,4,6,7,8,9,10,11,12,13,14$ & 1,5 & & 0.006 \\
1,5 & $4,5,8,9,10,11,12,13,14,15$ & 1,6 & & 0.006 \\
1,6 & $1,4,5,8,10,11,12,13,15$ & 1,7 & & 0.006 \\
1,7 & $12,3,4,6,7,8,9,10,11,12,13,14$ & 5,8 & 1,10 & 0.006 \\
1,8 & $1,4,5,8,10,11,12,13,14,15$ & 1,7 & 9 & 0.006 \\
1,9 & $1,2,3,4,7,8,10,11,12,13,14$ & 1,10 & & 0.006
\end{tabular}


fault coverage rate of $100 \%$, fault isolation rate of $71.1 \%$, the average diagnostic reasoning time is $0.006 \mathrm{~s}$

The experimental results show that the fault isolation rate reaches $100 \%$ for a single fault case and $71.1 \%$ for a double fault case. The fault coverage rate is $100 \%$ in both cases, and the running time of the diagnostic algorithm is acceptable Within the real-time diagnosis of the real requirements.

\section{Conclusion}

In this paper, the multi-signal model is used in the accusation system, which can describe the correlation between the fault module and test more quickly and accurately and give a more accurate correlation matrix. The diagnosis process is based on knowledge reasoning without focusing on a certain parameter test,and meets the diagnostic needs of the system level. The method obtained satisfactory results in the diagnosis of Apollo spacecraft before launch.

\section{References}

1. L.Q. Song, D. Song, J.W. Li. Comouter measurement and control,22:9-12(2014).

2. D. Somnath, K.R. Pattipati, R. Vijay,et al. IEEE Aerospace and Electronic Systems Magazine, 10: 14-25(1995)

3. K.R. Pattipati, V. Raghavan, M. Shakeri,et al. Proc of American Control Conference, 1989-1995(1994).

4. X. Gao, B. Long, X.J. Yang. CHINA MEASUREMENT \& TEST ,37: 49-53(2011).

5. C.T. Kuang. Zhengzhou University (2015).

6. K. Lian, B. Long, H.J. Wang.ACTA ARMAMENTARII, 29: 352-356(2008).

7. J. G. Wohl. IEEE Transactions on Systems, Man and Cybernetics, 12: 469-478(1982). 